\section{Allergische Rhinitis: Kortikoidspray ohne Rezept}

D ie Prävalenz der allergischen Rhinitis verdoppelt sich etwa alle acht Jahre. Prof. Dr. Ralph Mösges, Klinikum der Universität zu Köln, wies darauf hin, dass je nach untersuchter Population zwischen 30 und 50\% der Personen an dieser Erkrankung leiden. Interessanterweise erkranken auch immer mehr ältere Personen: „25\% der Erstmanifestationen eines Heuschnupfens treten nach dem 40. Lebensjahr auf", erläutert Mösges. Die Rhinitis allein sei nur die Spitze des Eisbergs. Hinter den Symptomen stehe meist eine schwelende Entzündung, so Mösges.

Die Patienten fühlen sich allgemein stark beeinträchtigt. Neben Müdigkeit und Schlafstörungen drohen auch verminderte Leistungsfähigkeit sowie sozialer Rückzug - die Lebensqualität ist deutlich beeinträchtigt. Umso schlimmer ist die Tatsache, dass zwei Drittel der Patienten mit der Behandlung unzufrieden sind und die Hälfte wegen dieser Erkrankung nicht mehr den Arzt aufsucht [Derebery J et al. Otolaryngol Head Neck Surg 2008; 139: 198-205].

Zur Selbstmedikation steht mittlerweile Beclomethason (z. B. Otriven Allergie $\left.\mathrm{Aktiv}^{\circledast}\right)$ zur Verfügung. Mösges betonte, dass nasale Steroide zu den Therapeutika der ersten Wahl bei intermittierender oder perennialer allergischer Rhinitis für Kinder und Erwachsene gehören (Leitlinie Allergische Rhinokonjunktivitis der Deutschen Gesellschaft für Allergologie und klinische Immunologie).

Wichtig ist, Glukokortikoide bereits beim ersten Pollenflug einzunehmen, unterstrich Mösges. Das Nasenspray sollte nicht nur bei Bedarf, sondern längere Zeit kontinuierlich angewendet werden. Der Vorteil der nasalen Kortikoide besteht neben der Linderung der lokalen Symptome auch in einem anhaltenden antiinflammatorischen Effekt. Damit kann eine sonst persistierende Entzündung und das "nasale Priming“ (zunehmende Empfindlichkeit der Nasenschleimhaut bei gleicher Allergenexposition) unterdrückt werden.

Dr. Andreas Fischer

Pressegespräch „Die allergische Rhinitis in der Selbstmedikation " anlässlich der 45. Fortbildungsveranstaltung HNO, Mannheim, 27. Oktober 2011 Veranstalter: Novartis Consumer Health, München

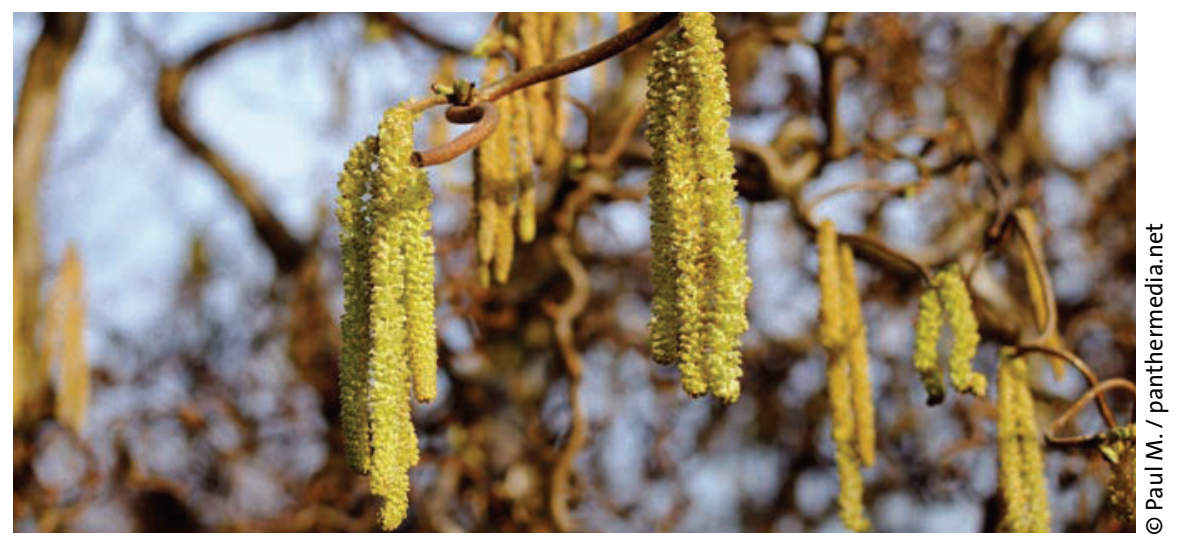

Bereits im Januar fliegen die ersten Pollen, z. B. die der Hasel - nicht zu früh also für Allergiker sich für die Saison zu rüsten.

\section{Spezifische Immuntherapie: Erinnern fördert Compliance}

m Februar hat das Wedeler Pharmaunternehmen ALK den „ALK-Compliance-Service“ eingeführt. Das Ziel dieses Dienstes ist es, Patienten, die eine spezifische Immuntherapie (SIT) erhalten, über den gesamten Therapiezeitraum von drei Jahren zu unterstützen und an die Arzneimitteleinnahme zu erinnern.

Erste Erfahrungen sechs Monate nach der Einführung des ALK-Compliance-Services zeigen, dass Patienten und Fachärzte von der Dienstleistung profitieren. „Der Compliance-Service wird von den Patienten gut angenommen. Wichtig ist auch: Meine Mitarbeiter werden deutlich entlastet, da das Recallprocedere weitestgehend entfällt", betonte Dr. Andreas Horn, Allergologe aus Heidelberg.

Ein Vorteil des ALK-ComplianceServices liegt in der einfachen Handhabung und der Bereitstellung des Services für alle Applikationsformen der spezifischen Immuntherapie: Bei der Erstverordnung füllt die Arzthelferin zusammen mit dem Patienten ein Bestellformular aus. Daraufhin erhält der Patient ein Compliancepaket per Post, das Patientenbroschüren zum Therapieverlauf sowie auf die Therapieform abgestimmte Compliance-Hilfen ent- hält. Hierbei haben alle Patienten die Möglichkeit, sich entweder per SMS oder per E-Mail an die Abholung des Folgerezeptes erinnern zu lassen. AuBerdem können sich Patienten, die eine sublinguale Immuntherapie erhalten, zusätzlich für einen täglichen Erinnerungsservice zur Arzneimitteleinnahme registrieren.

Ökonomisch verspricht sich Horn durch den Einsatz des Services über eine verbesserte Patienten-Compliance mittelfristig Kosteneinsparungen und somit eine höhere Akzeptanz bei den Kostenträgern und den Prüfgremien. ALK bietet den ALK-Compliance-Service für allergologisch tätige Ärzte und deren Patienten an.

red 